\title{
Magnetic Reconnection and Turbulent Mixing: From ISM to Clusters of Galaxies
}

\author{
Lazarian, A. \\ Dept. of Astronomy, UW-Madison, (lazarian@astro.wisc.edu) \\ Cho, J. \\ Dept. of Astronomy, UW-Madison, (cho@astro.wisc.edu)
}

\begin{abstract}
Magnetic reconnection, or the ability of the magnetic field lines that are frozen in plasma to change their topology, is a fundamental problem of magnetohydrodynamics (MHD). We briefly examine the problem starting with the well-known Sweet-Parker scheme, discuss effects of tearing modes, anomalous resistivity and the concept of hyperresistivity. We show that the field stochasticity by itself provides a way to enable fast reconnection even if, at the scale of individual turbulent wiggles, the reconnection happens at the slow Sweet-Parker rate. We show that fast reconnection allows efficient mixing of magnetic field in the direction perpendicular to the local direction of magnetic field. While the idea of stochastic reconnection still requires numerical confirmation, our numerical simulations testify that mixing motions perpendicular to the local magnetic field are up to high degree hydrodynamical. This suggests that the turbulent heat transport should be similar to that in non-magnetized turbulent fluid, namely, should have a diffusion coefficient $\sim V_{L} L$, where $V_{L}$ is the amplitude of the turbulent velocity and $L$ is the scale of the turbulent motions. We present numerical simulations which support this conclusion. The application of this idea to thermal conductivity in clusters of galaxies shows that this mechanism may dominate the diffusion of heat and may be efficient enough to prevent cooling flow formation.
\end{abstract}

Keywords: interstellar medium, intergalactic medium, magnetic fields

\section{Introduction}

Interstellar plasma has high conductivity and therefore magnetic field and fluid move together. As the result magnetic field influences fundamental properties of interstellar plasma and must be accounted in star formation processes, support and evolution of molecular clouds, transfer of mass and energy in the interstellar space etc. Magnetic fields control cosmic rays propagation, induce Parker (see Parker 1979) and magneto-rotational (see Balbus \& Hawley 1998) instabilities. Transport processes in the interstellar medium and beyond it, e.g. clusters of galaxies, depend on the ability of magnetic field lines to change their topology or to reconnect. It is difficult to overestimate the role of magnetic reconnection as it is also a necessary component of magnetic dynamo (see Parker 1979, and also Vishniac, Lazarian \& Cho 2003

(c) 2019 Kluwer Academic Publishers. Printed in the Netherlands. 
for a recent review), and it is likely to affect magnetic flux removal from hotbeds of star formation etc. More generally, it is impossible to claim that we understand MHD unless we can predict whether crossing magnetic flux tubes will reconnect or bounce from one another.

Mixing motions perpendicular to magnetic field lines are an essential part of the Goldreich-Sridhar model (1995, henceforth GS95) of MHD turbulence. Should those motions proceed in the hydrodynamic-type fashion or magnetic field would accumulate unresolvable knots that would affect the motion of the fluid? The answer to this question has far reaching consequences for heat transport in the interstellar medium and beyond. Indeed, magnetic fields are known to suppress heat conductivity (see Chandran \& Cowley 1998) in the direction perpendicular to B. Transport of heat along wondering magnetic field lines (Narayan \& Medvedev 2001, Zakamska \& Narayan 2002) partially alleviates the problem. If reconnection proceeds efficiently, the mixing motions should allow an efficient transport of heat in magnetized turbulent fluids. This would have big implications for problems from cooling flows in the clusters of galaxies (see Fabian 1994) to mixing layers in our and other galaxies (see Slavin, Shull, \& Begelman 1993).

In $\S 2$ and $\S 3$, we discuss reconnection and, in $\S 4$ and $\S 5$, we consider turbulent mixing of magnetized plasma.

\section{Sweet-Parker, Petschek Models and their Modifications}

The literature on magnetic reconnection is rich and vast (see, for example, Priest \& Forbes (2000) and references therein). We start by discussing a robust scheme proposed by Sweet and Parker (Parker 1957; Sweet 1958). In this scheme oppositely directed magnetic fields are brought into contact over a region of length $L_{x}$ (see Fig. 1). In general there will be a shared component, of the same order as the reversed component. However, this has only a minor effect on our discussion. The gradient in the magnetic field is confined to the current sheet, a region of vertical size $\Delta$, within which the magnetic field evolves resistively. The velocity of reconnection, $V_{r}$, is the speed with which magnetic field lines enter the current sheet, and is roughly $\eta \approx V_{r} \Delta$. Arbitrarily high values of $V_{r}$ can be achieved (transiently) by decreasing $\Delta$. However, for sustained reconnection there is an additional constraint imposed by mass conservation. The plasma initially entrained on the magnetic field lines must escape from the reconnection zone. In the Sweet-Parker scheme this means a bulk outflow, parallel to the field lines, within the current sheet. Since the mass enters along a zone of width $L_{x}$, and is 
ejected within a zone of width $\Delta$, this implies

$$
\rho V_{\text {rec }} L_{x}=\rho^{\prime} V_{A} \Delta
$$

where we have assumed that the outflow occurs at the Alfvén velocity $V_{A}$. This is actually an upper limit set by energy conservation. If we ignore the effects of compressibility $\rho=\rho^{\prime}$ and the resulting reconnection velocity allowed by Ohmic diffusivity and the mass constraint is

$$
V_{\text {rec,sweet-parker }} \approx V_{A} \mathcal{R}_{L}^{-1 / 2},
$$

where $\mathcal{R}_{L}$ is the Lundquist number using the current sheet length. Depending on the specific astrophysical context, this gives a reconnection speed which lies somewhere between $10^{-3}$ (stars) and $10^{-10}$ (the galaxy) times $V_{A}$.

Attempts to accelerate Sweet-Parker reconnection are numerous. We start by considering schemes to broaden the current sheet. Anomalous resistivity is known to broaden current sheets in laboratory plasmas. But this is microphysical broadening which is not important when the thickness of the current sheet that in the Sweet-Parker scheme scales as $L_{x}^{1 / 2}$ is much larger than the Larmor radius of the thermally moving proton. The latter scale is of the order of $200 \mathrm{~km}$ in the interstellar medium and it would be naive to expect to be able to squeeze the interstellar gas out through such a narrow slot (see eq (1).

Tearing modes are a robust instability connected to the appearance of narrow current sheets (Furth, Killeen, \& Rosenbluth 1963). Their importance for reconnection by many authors (see Strauss 1988, Zweibel 1989). The tearing modes broaden the reconnection layer and enhance the reconnection speed. A treatment of the problem in Lazarian \& Vishniac (1999; hereafter LV99) of 3D reconnection involving tearing modes provided an estimate

$$
V_{\text {rec,tearing }}=V_{A}\left(\frac{\eta}{V_{A} L_{x}}\right)^{3 / 10},
$$

which is substantially faster than the Sweet-Parker rate, but still very slow in any astrophysical context. Note that unlike anomalous effects, tearing modes do not require any special conditions and therefore should constitute a generic scheme of reconnection.

Hyperresistivity was introduced in the literature (see Hameiri \& Bhattacharjee 1987, Strauss 1988) to describe the ability of current sheet instabilities to drive plasma turbulence that would affect the reconnection rates. Tearing instability is one of the major current sheet instabilities and therefore the concept of hyperresistivity is related to the previously discuss issue. If the reconnecting magnetic fields are 
nearly anti-parallel, the turbulent mixing of magnetic field lines becomes possible and the reconnection can go much faster. However, this assumption of very nearly parallel field lines substantially limits the application of the concept.

The failure to find fast reconnection speeds due to broadening the current sheet has stimulated interest to fast reconnection through radically different global geometries. Petschek (1964) conjectured that reconnecting magnetic fields would tend to form structures whose typical size in all directions is determined by the resistivity (' $\mathrm{X}$-point' reconnection). This results in a reconnection speed of order $V_{A} / \ln \mathcal{R}_{L}$. However, attempts to produce such structures in numerical simulations of reconnection have been disappointing. Typically the X-point region collapses toward the Sweet-Parker geometry as the Lundquist number becomes large (Biskamp 1984, 1986, 1996; Wang, Ma, \& Bhattacharjee 1996; Ma \& Bhattacharjee 1996). ${ }^{1}$

One may invoke collisionless plasma effects to stabilize the X-point reconnection (for collisionless plasma). For instance, a number of authors (Shay et al. 1998; Shay \& Drake 1998; Shay et al. 1999) have reported that in a two fluid treatment of magnetic reconnection, a standing whistler mode can stabilize an X-point with a scale comparable to the ion plasma skin depth, $c / \omega_{p i} \sim\left(V_{A} / c_{s}\right) r_{L}$. The resulting reconnection speed is a large fraction of $V_{A}$, and apparently independent of $L_{x}$, which would suggest that something like Petschek reconnection emerges in the collisionless regime. However, these studies have not yet demonstrated the possibility of fast reconnection for generic field geometries, since they assume that there are no bulk forces acting to produce a large scale current sheet. Similarly, those studies do not account for fluid turbulence. Magnetic fields embedded in a turbulent fluid will give fluctuating boundary conditions for the current sheets. On the other hand, boundary conditions need to be fine tuned for a Petschek reconnection scheme (Priest \& Forbes 2000).

\section{Stochastic Reconnection}

The scheme of the stochastic reconnection is presented in Fig. 1. We consider the case in which there exists a large scale, well-ordered magnetic field, of the kind that is normally used as a starting point for discussions of reconnection. This field may, or may not, be ordered on the largest conceivable scales. However, we will consider scales smaller

\footnotetext{
${ }^{1}$ Recent plasma reconnection experiments (Yamada et al. 2000) do not support Petschek scheme either.
} 
than the typical radius of curvature of the magnetic field lines, or alternatively, scales below the peak in the power spectrum of the magnetic field, so that the direction of the unperturbed magnetic field is a reasonably well defined concept. In addition, we expect that the field has some small scale 'wandering' of the field lines. On any given scale the typical angle by which field lines differ from their neighbors is $\phi \ll 1$, and this angle persists for a distance along the field lines $\lambda_{\|}$with a correlation distance $\lambda_{\perp}$ across field lines (see Fig. 1). Unlike the traditional Sweet-Parker scheme it allows many magnetic field lines to enter simultaneously into the reconnection region. The reconnection therefore happens simultaneously at many surfaces and over substantially smaller scales. Therefore the reconnection velocity increases substantially. The limitation for the scheme is the outflow of the matter, which in this case is controlled by magnetic field line diffusivity. More discussion of the scheme is provided below.

Note that the model of stochastic reconnection is 3 dimensional $^{2}$. Our result cannot be obtained by considering two dimensional turbulent reconnection (cf. Matthaeus \& Lamkin 1985). In fact, it does not arise from the turbulent transport of magnetic flux, as it incorrectly understood in Kim \& Diamond (2001), but is a geometric effect arising from the appearance of stochastic field line wandering in three dimensions.

The modification of the mass conservation constraint in the presence of a stochastic magnetic field component is self-evident. Instead of being squeezed from a layer whose width is determined by Ohmic diffusion, the plasma may diffuse through a much broader layer, $L_{y} \sim\left\langle y^{2}\right\rangle^{1 / 2}$ (see Fig. 1), determined by the diffusion of magnetic field lines. This suggests an upper limit on the reconnection speed of

$$
V_{\text {rec, } \text { mass constr }} \sim V_{A}\left(\left\langle y^{2}\right\rangle^{1 / 2} / L_{x}\right)
$$

To make further progress one should adopt a model of magnetic turbulence. LV99 considered the whole range of possible models. Using GS95 scalings and assuming that the localized reconnection goes at the Sweet-Parker rate it is possible to get

$$
V_{\text {rec, }} S P-\text { Alfven, local } \sim V_{A}\left(\eta / V_{A} L\right)^{1 / 4}
$$

2 The Sweet-Parker scheme can easily be extended into three dimensions, in the sense that one can take a cross-section of the reconnection region such that the shared component of the two magnetic fields is perpendicular to the cross-section. In terms of the mathematics nothing changes, but the outflow velocity becomes a fraction of the total $V_{A}$ and the shared component of the magnetic field will have to be ejected together with the plasma. This result has motivated researchers to do most of their calculations in $2 \mathrm{D}$, which has obvious advantages for both analytical and numerical investigations. 

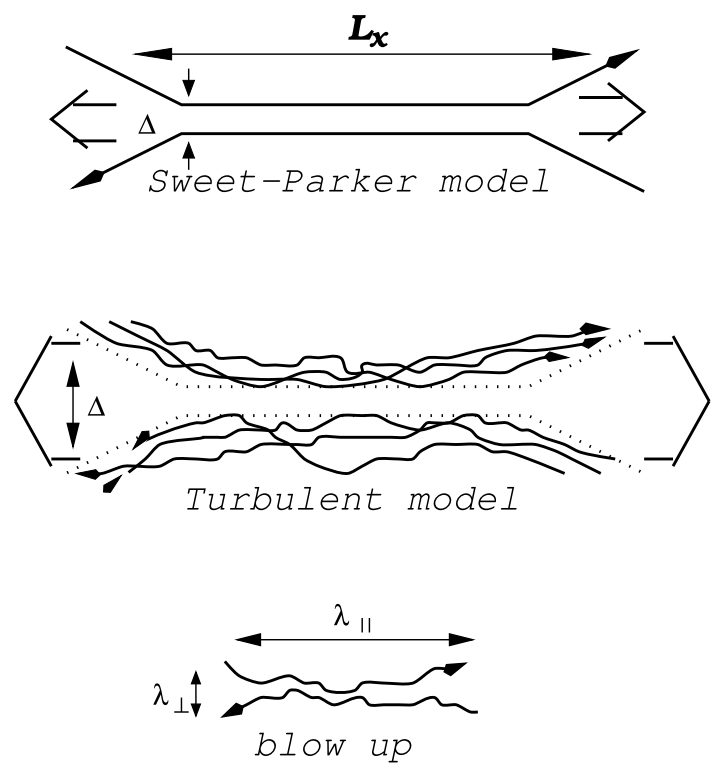

Figure 1. Upper plot: Sweet-Parker scheme of reconnection. Middle plot: scheme of stochastic reconnection that accounts for field line stochasticity. Lower plot: a blow up of the contact region. Thick arrows depict outflows of plasma. From Lazarian \& Vishniac (2000)

where the turbulent velocity at the scale $L$ is assumed to be Alfvenic. The isotropy of the fast modes has been proved numerically (Cho \& Lazarian 2002, also review by Cho \& Lazarian 2003). Our work suggests that both in magnetic pressure dominated and gas pressure dominated environments fast modes scaling is similar to that of acoustic turbulence. Assuming that the spectrum of such waves is truncated because of resistivity, LV99 obtained

$$
V_{\text {rec, }} \text { SP-fast, local } \sim V_{A}\left(\eta / V_{A} L\right)^{1 / 6},
$$

which is faster than the Alfvenic turbulence can provide.

If we assume that resistivity presents the bottleneck for the reconnection rate, the global reconnection rate can be estimated as $V_{\text {rec local }} L_{x} / \lambda_{\|}$. If the plasma is fully ionized $\lambda$ gets very small and the limit for the global reconnection rate provided by the resistivity scales as $V_{A} R m^{1 / 4} \gg$ $V_{A}$ for Alfven modes and $V_{A} R m^{1 / 2} \gg V_{A}$ for fast modes. Similarly, considering other possible bottlenecks, LV99 concluded that the mass conservation (eq. (4)) presents the most stringent constraint on the reconnection rate for the reconnection of the fully ionized gas. For Alfvenic turbulence this gives (see LV99)

$$
V_{\text {rec,global }} \approx V_{A} \min \left[\left(L_{x} / l\right)^{1 / 2},\left(l / L_{x}\right)^{1 / 2}\right]\left(V_{L} / V_{A}\right)^{2}
$$


The case of the partially ionized gas is considered in Lazarian, Vishniac, \& Cho (2003). There it is found that in the assumption of the local Sweet-Parker rates, the reconnection is slower because the turbulent cascade gets modified by ambipolar drag. However, the resulting reconnection rates are sufficiently high to be important for removing flux from star-forming clouds.

\section{Mixing and Turbulent Diffusion in Magnetized Media}

Let us start with a note of warning. The scheme of stochastic reconnection that we have discussed is very different from the so-called "turbulent diffusivity of magnetic field". The latter is a wrong concept that was used at earlier stages of the mean dynamo theory (see recent review by Vishniac, Lazarian \& Cho 2003) to justify some of its assumptions. According to "turbulent diffusivity" idea, the magnetic fields of opposite polarity can be mixed up by turbulence and then dissipate at small scales. This picture is wrong as magnetic fields are dynamically important and they get to dominate the small scale motions of the magnetized fluid (see Parker 1992). In the stochastic reconnection scheme magnetic field wondering happens within each layer of the opposite polarity, but no small scale mixing of the magnetic field of opposite polarities is assumed.

Mixing of magnetic field lines happens in the direction perpendicular to local direction of the magnetic field. GS95 picture of turbulence can be understood in terms of the eddies in the planes perpendicular to magnetic field lines (see discussion in Cho, Lazarian \& Vishniac 2002). LV99 showed that the eddies will not be forming magnetic knots if the reconnection is as fast as the stochastic reconnection scheme suggests. This means that the motions of the magnetized fluid will be very similar to the hydrodynamic motions in the planes perpendicular to the local direction of magnetic field.

Cho, Lazarian \& Vishniac (2002) scrutinized the statistics of the motions of the magnetized turbulent fluid in the direction perpendicular to the local direction of magnetic field. Using numerical simulations they found that for sufficiently strong magnetic field the turbulent motions perpendicular to magnetic field are identical to the hydrodynamic motions. Although this fact cannot be used as a proof of the stochastic reconnection but it can be used as the evidence that, in the absence of any anomalous effects, the change of magnetic topology does not constrain fluid motions.

While the "turbulent diffusivity of magnetic field" is a faulty concept, turbulence may provide efficient diffusivity for heat and matter. 

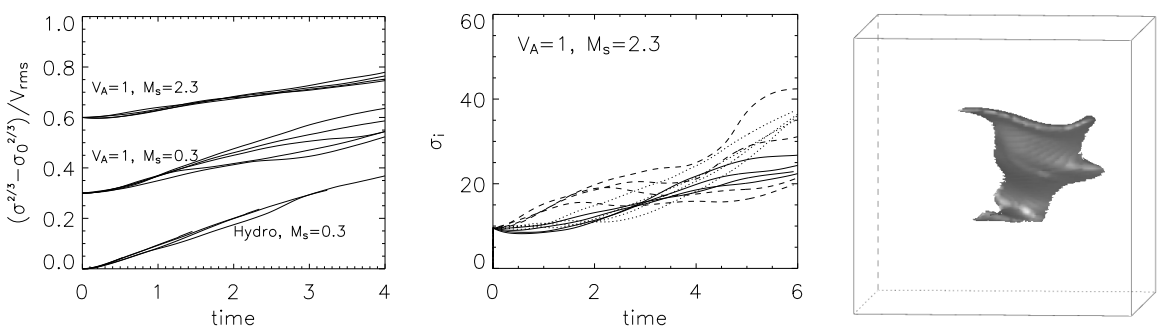

Figure 2. Left: $\left(\sigma^{2 / 3}-\sigma_{0}^{2 / 3}\right) / V_{L}$ vs. time. $\sigma$ is the standard deviation of the passive scalar field. Y-axis is in the unit of box-size and Y-values are shifted by 0.3 units for convenience. Note that the slope does not strongly depend on the mean field $B_{0}$ or sonic Mach number $M_{s}$. Middle: $\sigma_{i}(\mathrm{i}=\mathrm{x}, \mathrm{y}$, and $\mathrm{z})$ vs. time. Solid lines=parallel to $\mathbf{B}_{0}$; dashed and dotted lines=perpendicular to $\mathbf{B}_{0}$. Right: Snapshot of the passive scalar field for $V_{A}\left(\equiv B_{0} / \sqrt{4 \pi \rho}\right) \sim V_{L}$ and $M_{s}=0.3$. $\mathbf{B}_{0}$ is parallel to the dashed line (i.e. vertical). Turbulent motions provide efficient mixing of the passive scalar. Left and Middle panels suggest that magnetic field does not suppress this turbulent mixing process. Obviously, when $V_{A}$ is lower than $V_{L}$, the efficiency of turbulent mixing is no less than that in the $V_{A} \sim V_{L}$ case. Results are obtained from using $192^{3}$ and $216^{3}$ grid points.

Indeed, the turbulent motions perpendicular to magnetic field should transport heat and other passive scalar field in the same way as the hydrodynamic turbulence does. Below we will be mostly talking about heat transfer, as this is the problem of largest astrophysical significance. The corresponding diffusion coefficient is expected to be of the order of $L V_{L}$.

Do we expect the turbulent heat transport to be strongly anisotropic? As the large scale field wanders, the direction of the local magnetic field that determines the mixing motions of hydrodynamic-like eddies changes as well. Therefore no strong anisotropy in heat conduction is expected.

To test our theoretical considerations related to the diffusion of heat we performed numerical simulations using our hybrid ENO MHD code described, for instance, in Cho \& Lazarian (2002). We use a passive scalar $\psi(\mathbf{x})$ to trace thermal particles. We inject a passive scalar with a Gaussian profile:

$$
\psi\left(\mathbf{x}, t=t_{0}\right) \propto \exp ^{-\left(\mathbf{x}-\mathbf{x}_{0}\right)^{2} / \sigma_{0}^{2}}
$$

where $\sigma_{0}=1 / 16$ of a side of the numerical box and $\mathbf{x}_{0}$ lies at the center of the computational box. The value of $\sigma_{0}$ ensures that the scalar is injected in the inertial range of turbulence. The scalar field follows the continuity equation: $\partial \psi / \partial t+\nabla \cdot(\psi \mathbf{v})=0$.

In Figure 2, we compare time evolution of $\sigma$ in hydrodynamic case and in MHD cases. The results are very close and this enables us to 
suggest that the coefficient of thermal diffusion is

$$
\kappa_{\text {dynamic }}=C_{d y n} L V_{L},
$$

where $C_{d y n}$ is a constant of order unity, $V_{L}$ is the amplitude of the r.m.s. turbulent velocity, and $L$ is the scale of the turbulent motions. This is the effective diffusion by turbulent motions suitable for scales larger than injection scale $L$. The value of $C_{d y n}$ remains almost constant for $V_{A}$ 's of up to the equipartition value $V_{A} \sim V_{L}$. The exact value of

$C_{d y n}$ is uncertain. In hydrodynamic cases, $C_{d y n}$ is of order of $\sim 0.3$ (see Lesieur 1990 chapter VIII and references therein).

\section{Astrophysical Implications}

In the sections above we discussed two issues: magnetic stochastic reconnection and turbulent transport of heat through mixing motions. We believe that the two processes are intrinsically connected and fast reconnection implies efficient turbulent transport. Nevertheless, while testing of stochastic reconnection is still a challenging problem, efficient mixing and heat transport has been already tested numerically.

The astrophysical implications of stochastic reconnection were discussed in LV99 where it was shown that it naturally accounts for Solar flares, for operation of galactic dynamo, etc. In Lazarian et al. (2003), its implications for gamma ray busts were analyzed, however. This does not limit the list of the possible applications. For instance, it is easy to see that stochastic reconnection can provide efficient removal of magnetic flux from starforming clouds. A proper discussion of these implications requires a more extended review. Here we briefly consider implications of fast turbulent transport.

\section{Clusters of Galaxies}

It is widely accepted that ubiquitous X-ray emission due to hot gas in clusters of galaxies should cool significant amounts of intracluster medium and this must result in cooling flows (Fabian 1994). However, observations do not support the evidence for the cool gas (see Fabian et al. 2001) which is suggestive of the existence of heating that replenishes the energy lost via X-ray emission. Heat transfer from the outer hot regions can do the job, provided that the heat transfer is sufficiently efficient.

Gas in the clusters of galaxies is magnetized and the conventional wisdom maintains that the magnetic fields strongly suppress thermal conduction perpendicular to their direction. Realistic magnetic fields are turbulent and the issue of the thermal conduction in such a situation 
has been long debated. A recent paper by Narayan \& Medvedev (2001) obtained the estimates for the thermal conductivity of the turbulent magnetic fields.

However, Narayan \& Medvedev (2001) treated the turbulent magnetic fields as static. In hydrodynamical turbulence it is possible to neglect plasma turbulent motions only when the diffusion of electrons which is the product of the electron thermal velocity $v_{\text {elect }}$ and the electron mean free path in plasma $l_{m f p}$, i.e. $v_{\text {elect }} l_{m f p}$, is greater than the turbulent velocity $V_{L}$ times the turbulent injection scale $L$, i.e. $L V_{L}$. If such scaling estimates are applicable to heat transport in magnetized plasma, the turbulent heat transport should be accounted for heat transfer within clusters of galaxies. Indeed, data for $l_{m f p} v_{\text {elect }} l_{m f p}$ given in Zakamska \& Narayan (Narayan \& Medvedev 2001) provide the diffusion coefficient $\kappa_{S p} \equiv v_{\text {elect }} l_{m f p} \sim 6.22 \times 10^{30} \mathrm{~cm}^{2} \mathrm{sec}^{-1}$ (for Hydra A). If turbulence in the cluster of galaxies is of the order of the velocity dispersion of galaxies, while the injection scale $L$ is of the order of $20 \mathrm{kpc}$, the turbulent diffusion coefficient is $\kappa_{\text {dynamic }} \sim L V_{L} \sim 6 \times 10^{30}$ $\mathrm{cm}^{2} \mathrm{sec}^{-1}$, where we take $V_{L} \sim 1000 \mathrm{~km} / \mathrm{sec}$.

Although the estimates for the electron thermal diffusivity and our estimate of the turbulent diffusivity are of the same order the applicability of Narayan \& Medvedev's model is a bit restricted - their model requires strong (i.e. $B_{0} \sim \delta B$ ) mean magnetic field. While there are strong mean magnetic fields in the Galaxy, this is unlikely for the intracluster medium. When the mean field is weak, turbulence at the scales smaller than the characteristic magnetic field scale $\left(\equiv l_{B}\right)$ will follow the GS95 model. Therefore, Narayan \& Medvedev's model is fine for the scale of the characteristic magnetic field scale, which is a bit smaller than energy injection scale of turbulence. But, at the scales larger than the characteristic magnetic field scale, the field line diverges according to random walk. Then, the efficient diffusivity is $\sim \kappa_{S p}\left(l_{B} / R\right)^{2}$, where, $R$ is the size of the system. However, our turbulent mixing model still gives the same $\kappa_{\text {dynamic }}$ on the scales larger than $l_{B}$. Therefore, our model has a broad range of applications.

\section{Local Bubble and SNRs}

Now let us compare the results for thermal diffusivity for other important astrophysical situations. The Local Bubble $(\mathrm{LB})$ is a hot $(T \sim$ $\left.10^{6} \mathrm{~K} ; k T \sim 100 \mathrm{eV}\right)$, tenuous $\left(n \sim 0.008 / \mathrm{cm}^{3}\right)$ cavity immersed in the interstellar medium (Berghofer et al 1998; Smith \& Cox 2001). Turbulence parameters are uncertain. We take typical interstellar medium values: $L \sim 10 \mathrm{pc}$ and $V_{L} \sim 5 \mathrm{~km} / \mathrm{sec}$. For these parameters, the ratios of $\kappa_{\text {dynamic }}$ to $\kappa_{S p}$ are

$$
\mu_{\text {in }}=\kappa_{\text {dynamic }} / \kappa_{S p} \sim 0.05, \quad \text { (inside LB) }
$$




$$
\mu_{\text {mix }}=\kappa_{\text {dynamic }} / \kappa_{S p} \sim 100, \quad(\text { in mixing layer }),
$$

where we take $\bar{T} \sim \sqrt{T_{c} T_{h}} \sim 10^{5} K, \bar{n} \sim \sqrt{n_{c} n_{h}} \sim 0.1 / \mathrm{cm}^{3}$ (Begelman \& Fabian 1990), $T_{c} \sim 10^{4} \mathrm{~K}, n_{c} \sim 1 / \mathrm{cm}^{3}, T_{h} \sim 10^{6} \mathrm{~K}$, and $n_{h} \sim$ $0.008 / \mathrm{cm}^{3}$. Subscripts ' $\mathrm{h}$ ' and 'c' stand for 'hot' and 'cold', respectively. This implies that thermal conduction along static magnetic field lines is suppressed when electrons gyrate through the 'cold' and 'dense' mixing layer. Note that turbulent diffusion is still effective in the mixing layer. We expect similar results for supernova remnants since parameters are similar.

\section{Summary}

In the article above we briefly reviewed a number of existing ideas about reconnection. Analysis of the solar and other reconnection-related data provides a number of requirements to the successful candidate. It should be robust to act in the situation when the boundary conditions constantly change due to turbulence. It should typically provide reconnection rates of the order of one tenth $V_{A}$, but sometimes it should be much slower to allow the accumulation of the magnetic flux. Our analysis shows that the stochastic reconnection satisfies naturally to all these criteria.

Stochastic reconnection is different from the "turbulent magnetic diffusivity" idea. It does not require fine mixing of the magnetic fields

of opposite polarity. However, we show that the turbulent transport of heat happens in the presence of magnetic field very similarly to how it happens in pure hydrodynamics. In particular, we show that turbulent transport of heat in the intracluster matter is at least as fast as the earlier estimates based on the electron thermal conductivity suggested.

\section{Acknowledgements}

A.L. acknowledges the support of NSF Grant AST-0125544. The study of turbulent diffusion was done in collaboration with P. Moin and A. Honein at the Center for Turbulence Research in Stanford University as a part of Visiting Scholar Program in Summer 2002.

\section{References}

Balbus, S. A. and J. F. Hawley. Rev. Mod. Phys., 70, 1, 1998

Begelman, M. and A. Fabian. MNRAS, 244, 26, 1990 
Berghöfer, T. W., S. Bowyer, R. Lieu, and J. Knude. Astrophys. J., 500, 838, 1998

Biskamp, D. Phys. Lett. A, 105, 124, 1984

Biskamp, D. Phys. Fluids, 29, 1520, 1986

Biskamp, D. Astrophys. \& Sp. Sci., 242, 165, 1996

Chandran, B. and S. Cowley. Phy. Rev. Lett., 80, 3077, 1998

Cho, J. and A. Lazarian. Phy. Rev. Lett., 88(24), 245001, 2002

Cho, J. and A. Lazarian. astro-ph/0301462, 2003

Cho, J., A. Lazarian, and E. T. Vishniac. Astrophys. J., 564, 291, 2002

Fabian, A. C. Ann. Rev. Astro. \& Astrophys., 32, 277, 1994

Fabian, A. C., R. F. Mushotzky, P. E. J. Nulsen, and J. R. Peterson. MNRAS, 321, L20, 2001

Furth, H. P., J. Killeen, and M. N. Rosenbluth. Phys. Fluids, 6, 459 (1963)

Goldreich, P. and S. Sridhar. Astrophys. J., 438, 763, 1995 (GS95)

Hameiri, E. and A. Bhattacharjee. Phys. Fluids, 30, 1743, 1987

Kim, E.-J. and P. H. Diamond. Astrophys. J., 556, 1052, 2001

Lazarian, A., V. Petrosian, H. Yan, and J. Cho. astro-ph/0301181, 2003

Lazarian, A. and E. T. Vishniac. Astrophys. J., 517, 700, 1999 (LV99)

Lazarian, A. and E. T. Vishniac. Revista Mexicana de Astronomia y Astrofisica, $9,55,2000$

Lazarian, A., E. T. Vishniac, and J. Cho. Astrophys. J., submitted, 2003

Lesieur, M. Turbulence in fluids : stochastic and numerical modelling, 2nd. rev. ed., Kluwer Academic Publishers, Dordrecht, 1990

Ma, Z. W. and A. Bhattacharjee. J. Geophys. Res.. 101, 2641, 1996

Matthaeus, W. H. and S. L. Lamkin. Phys. Fluids, 28, 303, 1985

Narayan, R. and M. V. Medvedev. Astrophys. J., 562, L129, 2001

Parker, E. N. J. Geophys. Res., 62, 509, 1957

Parker, E. N. Cosmical Magnetic Fields, Clarendon Press, Oxford, 1979

Parker, E. N. Astrophys. J., 401, 137,1992

Petschek, H. E. 'Magnetic Field Annihilation', In W.H. Hess, editor, The Physics of Solar Flares, pages 425-439. NASA Special Pub. 50, Washington, DC, 1964

Priest, E. and T. Forbes. Magnetic Reconnection: MHD Theory and Applications (Cambridge: Cambridge University Press 2000)

Shay, M. and J. Drake. Geophys. Res. Lett., 25(20), 3759, 1998

Shay, M., J. Drake, R. Denton, and D. Biskamp. J. Geophys. Res., 103, 9165, 1998

Shay, M., J. Drake, B. Rogers, and R. Denton. Geophys. Res. Lett., 26(14), 2163, 1999

Slavin, J. D., J. M. Shull, and M. C. Begelman. Astrophys. J., 407, 83, 1993

Smith, R. and D. Cox. Astrophys. J. Supp., 134, 283, 2001

Strauss, H. R. Astrophys. J., 326, 412, 1988

Sweet, P. A. 'The Neutral Point Theory of Solar Flares', In B. Lehnert, editor, IAU Symp. 6, Electromagnetic Phenomena in Cosmical Plasma, pages 123-134. Cambridge Univ. Press, New York, 1958

Vishniac, E. T. and A. Lazarian. Astrophys. J., 511, 193, 1999

Vishniac, E. T., A. Lazarian and J. Cho. In T. Passot \& E. Falgarone, editors, Simulations of magnetohydrodynamic turbulence in astrophysics, Springer-Verlag, Lecture Notes in Physics, astro-ph/0205286, 2003

Wang, X., Z. W. Ma, and A. Bhattacharjee. Phys. Plasmas, 3(5), 2129, 1996

Yamada, M., H. Ji, S. Hsu, T. Carter, R. Kulsrud, and F. Trintchouk. Phys. Plasmas, $7(5), 1781,2000$

Zakamska, N. L. and R. Narayan. astro-ph/0207127, 2002

Zweibel, E. Astrophys. J., 343, 994, 1989 